\title{
Morphological and Biological Properties of a Strain of Chromogenic Acid-fast Bacteria and of its Phage-immune Variant
}

\author{
By EDITH MANKIEWICZ \\ Royal Edward Laurentian Hospital and McGill University, \\ Montreal, Canada
}

(Received 26 June 1960)

\begin{abstract}
SUMMARY
Phage-immune variants were detected among chromogenic acid-fast mycobacteria by the action of Mycobacterium phlei bacteriophage. One of the strains thus obtained showed not only phage immunity, but also a marked difference in colonial appearance, pigmentation, antigenic composition and pathogenicity for laboratory animals as compared with the original strain.
\end{abstract}

\section{INTRODUCTION}

During a recent survey of the phage susceptibility of chromogenic acid-fast bacteria isolated from patients with tuberculous disease, it was found that twentytwo out of ninety-eight of these strains were affected by exposure to one or more of the six phages used. In nine instances phage-immune dissociates were isolated from hazy plaques or from isolated colonies within clear plaques. Several of the phageimmune bacteria showed some morphological differences from the parent strain. In one case, the differences were so marked that this strain became the subject of the present study.

\section{METHODS}

Source of the strain of Mycobacterium. The chromogenic strain of acid-fast bacterium 41308 was isolated in 1956 from the sputum of a patient who, 26 months before, presented radiological evidence of widespread infiltration with bilateral cavitation. Typical Mycobacterium tuberculosis was isolated from the sputum at that time and twice again during the first 2 months of treatment. The patient received streptomycin, isonicotinic acid hydrazide and $p$-aminosalicylic acid for 2 years. Because the tuberculous disease appeared to be 'arrested', chemotherapy was suspended. Two months later, pure cultures of chromogenic acid-fast bacteria were recovered from the sputum.

Source of bacteriophages. The phages used were derived from Mycobacterium phlei, $M$. friburgensis, $M$. rabinowitsch, $M$. smegmatis and were received from Dr E. Vandra, Budapest, Hungary, and also phages D28 and D 29 received from Dr S. Froman, Olive View, California, U.S.A.

Characters of the chromogenic acid-fast bacterium and phage-immune variant studied: (a) cultural requirements and colonial appearance; (b) microscopic examination of the bacterial elements; $(c)$ comparison of their antigenic composi- 
tions by agar diffusion precipitation tests; $(d)$ susceptibility to antituberculosis agents; $(e)$ virulence tested by the neutral red cytochemical reaction and animal inoculation.

\section{RESULTS}

Strain 41308 produced smooth light-yellow colonies on egg-yolk media after incubation periods of 10-12 days at $37^{\circ}$, and of 16-18 days at $22^{\circ}(\mathrm{Pl}$. 1, fig. 1$)$. The pigment was not affected by light, and the strain was thought to belong to group III of Runyon's classification (Runyon, 1959). According to agar diffusion precipitation studies (Mankiewicz, 1958) this strain belonged to a group of about $6 \%$ of acidfast bacteria which show a close antigenic relationship with virulent tubercle bacilli. When this strain was spot tested with Mycobacterium phlei phage according to the technique described by Froman \& Bogen (1954) and modified by Takeya, Yoshimura, Tamamura \& Toda (1959), a slightly hazy plaque of regular contour was obtained. The subcultures of the phage-immune bacteria showed smooth colonies of darker pigmentation than those of the parent strain. Both strains grew only on media designed for $M$. tuberculosis.

When $\mathbf{0 . 2} \mathrm{ml}$. of the Mycobacterium phlei phage filtrate (referred to as phage $\mathbf{M}$ ) containing $10^{8}$ phage particles $/ \mathrm{ml}$. were added to $4.8 \mathrm{ml}$. of a 48 -hr. culture of strain 41308 in Tween albumin medium, subcultures made during the first 2 weeks on egg-yolk medium yielded either the original light-yellow colonies, or the more darkly pigmented colonies described above. After 2 weeks fewer colonies of a different aspect appeared on subculture. These colonies of a phage-immune variant were umbilicated or wart-like and at $37^{\circ}$ their pigment was orange. They lowered the $\mathrm{pH}$ value of the medium containing malachite green (Pl. 1, fig. 2). These growth characteristics were maintained through fifteen subcultures on egg-yolk medium at $37^{\circ}$. By maintaining the above-described conditions of incubation with phage $\mathbf{M}$, the phage-immune variant was isolated on several occasions from single colonies of strain 41308 which were selected after repeated plating and inoculated into liquid Tween albumin medium.

Besides the difference in the colonial aspect, the phage-immune variant grew on egg-yolk medium in 3 days at $37^{\circ}$, and in 5 days at $22^{\circ}$. It grew readily on nutrient agar and in brain-heart infusion. On agar medium, the colonies resembled those of Nocardia: they were lighter in colour than those grown on egg-yolk media, and were dry and granular. In brain-heart infusion, the variant produced a granular deposit at the bottom of the tube, and a veil at the surface of the liquid. Like certain Nocardia spp., it was able to use paraffin as sole carbon source. In contrast to the parent strain, the pigment of the variant was affected by light. At room temperature and in the light, only a light pigment was formed; the colonies were small, granular and closely resembled those of Mycobacterium tuberculosis (Pl. 1, fig. 3).

On microscopic examination, strain 41308 showed slender granulated rods. Approximately $80 \%$ of the bacilli of a 10-day culture in Tween albumin medium were acid-fast. Under the same conditions only $10 \%$ of the slender granulated phage-immune bacteria were acid-fast. There were no differences in the susceptibilities of the two strains to streptomycin or isonicotinic acid hydrazide (INH); both strains were susceptible to $1 \mu \mathrm{g}$. streptomycin $/ \mathrm{ml}$. and resistant to $10 \mu \mathrm{g}$. INH/ml. However, there was a significant difference in their susceptibility to ethionamide 
(TH 1314), the original strain being sensitive to $0.5 \mu \mathrm{g} . / \mathrm{ml}$. while the phage-resistant strain was resistant to $10 \mu \mathrm{g} . / \mathrm{ml}$. of this antituberculosis drug.

The most remarkable difference between strain 41308 and its variant was that the latter was lysogenic. Culture filtrates of the phage-immune strain lysed the parent strain 41308. In order to eliminate the possibility that the lysis was due to adsorbed phage, single colonies of the variant were transferred five times on egg-yolk media. One colony of the last culture was selected as inoculum for Dubos broth basal medium containing Tween 80, which is known to interfere, in the absence of albumin, with the adsorption of phages to bacteria (White \& Knight, 1958). Filtrates from the Dubos broth basal culture lysed the same mycobacteria as did phage M, namely, Mycobacterium phlei, M. stercoides, $M$. friburgensis, strain 41308 and five out of twelve test strains of unnamed chromogenic bacteria. The lytic spectrum suggested that the lysogenic variant carries a prophage similar to, or identical with phage $\mathrm{M}$.

Froman et al. (1955) reported that BCG strains did not show as high and as consistent a susceptibility to mycobacteriophages as did strains $\mathbf{H 3 7} \mathbf{R v}$ and H 37 Ra. Takeya et al. (1959) found that attenuated strains of human tubercle bacilli of Imamura were less susceptible to phage action than the original virulent strains. The attenuated strains, especially those of Imamura, showed changes in colonial appearance which were similar to those undergone by the variant of 41308. A study of the antigenicity and pathogenicity of this variant was attempted as follows.

(a) The agar diffusion precipitation technique (Mankiewicz, 1958) was used to study the antigenic composition of the variant. It was found that the precipitation pattern had changed from that of the original strain. The variant shared antigens with Mycobacterium phlei, with Nocardia asteroides and with other chromogenic acid-fast bacteria to a much larger extent than with virulent tubercle bacilli.

(b) The cytochemical reaction with neutral red (Dubos \& Middlebrook, 1948) was used with the following results:

$\begin{array}{lc}\text { Strain } 41308 & + \\ \text { Its phage-immune variant } & - \\ \text { Controls } & \\ \quad \text { H37 Rv } & ++ \\ \text { BCG } & ( \pm)\end{array}$

(c) Animal experiments. Five guinea-pigs (250 g.) were inoculated intramuscularly with $1 \mathrm{mg}$. wet weight of strain 41308, and five others with the same weight of the variant. After 4 and 8 weeks, the animals inoculated with 41308 gave strongly positive reactions to intradermal Old Tuberculin, whereas the guinea-pigs inoculated with the variant remained tuberculin-negative throughout the experiment. After 9 weeks, the latter did not show any sign of disease at autopsy. The animals inoculated with the original strain showed enlarged regional lymph nodes. However, liver and lungs were free from disease and no acid-fast bacteria were isolated from these tissues.

Forty Swiss mice (Rockefeller strain; $20 \mathrm{~g}$.) were inoculated intravenously with $0.5 \mathrm{mg}$. each of strain 41308; and forty others with the same amount of the variant organism. The animals were killed after 6 weeks. Thirty-two of the mice inoculated with the original strain showed macroscopic lesions in the lungs. None was observed 
in the animals inoculated with the variant strain. Homogenates of the right lungs and of the right kidneys of the eighty animals were inoculated on egg-yolk medium. Chromogenic, smooth-growing acid-fast bacteria were recovered from cultures of the kidneys (twelve instances) and from the lungs (twenty-six instances) from animals inoculated with strain 41308 . No bacteria were recovered from the forty kidney and forty lung cultures of the mice inoculated with the variant (Table 1).

Table 1. Comparison between the chromogenic acid-fast bacterium 41308 and its phage-immune variant

\author{
Growth on egg-yolk medium \\ At $37^{\circ}$ in the dark: \\ Matures in: \\ At $22^{\circ}$ in the light: \\ Matures in: \\ Growth on nutrient agar: \\ Matures in:
}

Susceptibility to ethionamide:

Neutral red reaction:

Sensitization of guinea-pigs to

Old Tuberculin:

Virulence for mice :

Lysogenicity:

Susceptibility to

Phage M:

Culture filtrate of variant:

Strain 41308
Yellow, smooth,
10-12 days
Yellow, smooth,
16-18 days
No growth
Sensitive
+
+
+
+
+
+

\section{DISCUSSION}

The questions which arise concern the origin and nature of the variant. An attempt may be made to narrow down the possibilities which appear to be as follows:

(1) Culture 41308 when isolated from the patient was 'mixed', containing phageimmune and phage-susceptible bacteria of different morphology.

(2) The phage-immune variant is the result of a spontaneous mutation. In both instances, phage $\mathbf{M}$ would have acted as a selective agent for the dissociate.

(3) Strain 41308 is an undetected or defective lysogenic strain.

(4) The change was phage-induced by $(a)$ the process of transduction, or $(b)$ by lysogenization.

The possibilities that culture $\mathbf{4 1 3 0 8}$ was mixed, or that the variant resulted from spontaneous mutation, can be disregarded because extensive plating of the original strain on egg-yolk medium never produced the faster growing umbilicated variant. Without previous exposure to phage $M$, no growth was produced by 41308 on nutrient agar, or in Youman's medium with serum containing $10 \mu \mathrm{g}$. ethionamide/ ml. Strain 41308 did not produce any lysis of strains selected at random. No lysis occurred within this strain after exposure to ultra-violet irradiation. It did not show any immunity to the lytic action of culture filtrates of the variant.

The changes described as characteristic for the variant were induced by phage $M$ only. To establish that the process at work was that of transduction, one would have to know the donor bacterium on which the phage had multiplied. The properties acquired by the variant of 41308 (umbilicated colonial morphology, the particular pigment, resistance to ethionamide, lysogenicity) do not permit tying the variant to 
Mycobacterium phlei, $M$. stercoides, $M$. friburgensis or to any of the acid-fast bacteria which were found to be susceptible to phage M. Although the possibility of transduction cannot be completely excluded, lysogenization appears the most plausible mechanism for the production of the variant.

The ability to produce phage and immunity are the most important changes due to lysogenization. Others have been described which concern the morphology and antigenicity of lysogenic bacteria. Ionesco (1953) observed that, due to the presence of prophage, the normally smooth colonies of Bacillus megaterium became rough and wart-like. Antigenic changes were described by Uetake, Nakagawa \& Akiba (1955), Uetake, Luria \& Burrows (1958), Staub, Tinelli, Luderitz \& Westphal (1959), Stocker (1958) and Zinder(1957) in Salmonellae after they became hosts to prophages. In Mycobacterium no naturally occurring lysogenic strains have been detected so far. Takeya et al. (1959) found that a phage-immune mutant of Mycobacterium sp. ATCC 607, resistant to phages A-2 and A-4, was lysogenic.

A connexion between the chromogenicity of colonies of mycobacteria and their immunity to phagolysis has been shown for Mycobacterium smegmatis and M.phlei (Bassermann, 1959); the phage-immune mutants showed an increase in pigmentation of their colonies.

While extensive studies have been devoted to the localization and chemical nature of the phage receptor groups of phage-susceptible Escherichia coli and various salmonellas, little is known about phage receptors in mycobacteria. Penso (1955) suggested that in mycobacteria there exists a close relationship between antigenantibody reaction and phage susceptibility. Tuberculo-antigens and phage receptors might be of similar molecular structure. The variant of strain 41308 showed that the loss of phage-susceptibility was linked with changes in the antigenic composition as demonstrated by the precipitation studies and the animal experiments. It is hoped that future studies will lead to a definition of the phage-affected areas in mycobacteria.

The colour plate which accompanies this communication was made possible through funds given to this Hospital in memory of the late Hazel Rexford Burke. I gratefully acknowledge this gift.

\section{REFERENCES}

Bassermann, F. J. (1959). Ergebnisse und Probleme der Mykobakteriophagenforschung. Beitr. Klin. Tuberk. 121, 185.

Dubos, R. J. \& Mrpdlebrook, G. (1948). Cytochemical reaction of virulent tubercle bacilli. Amer. Rev. Tuberc. 58, 698.

Froman, S. \& Bogen, E. (1954). Bacteriophage active against $M$. tuberculosis. I. Isolation and activity. Amer. J. Publ. Hlth, 44, 1326.

Froman, S., Will, D. W., Bliss, A., Conde, J. L., Krasnow, I. \& Bogen, E. (1955). Bacteriophage susceptibility and cultural characteristics of BCG and other tubercle bacilli. Dis. Chest, 28, 377.

Ionesco, H. (1953). Sur une propriété de B. megaterium liée à la présence d'un prophage. C.R. Soc. Biol., Paris, 237, 1794.

MaNkIewicz, E. (1958). Agar diffusion precipitation and complement fixation tests as applied to the study of the antigenic relationships between chromogenic acid-fast bacteria and M. tuberculosis. Canad. J. Microbiol. 4, 565.

Penso, G. (1955). Cycle of phage development within the bacterial cell. Protoplasma, 45, 251. 
Runyon, E. H. (1959). The recognition and characterization of pulmonary mycobacterial pathogens other than tubercle bacilli: a review. Bull. int. Union Tuberc. 39, 397.

Staub, A. M., Tinelli, R., Luderitz, O. \& Westphal, O. (1959). V. Rôle de quelques sucres, et en particulier des 3-6 didésoxyhexoses, dans la spécificité des antigènes $0 \mathrm{du}$ tableau de Kauffmann-White. Ann. Inst. Pasteur, 96, 303.

Stocker, B. A. D. (1958). Lysogenic conversion by the A phages of Salmonella typhimurium. J. gen. Microbiol. 18, ix.

Takeya, K., Yosimura, T., Tamamura, K. \& Toda, T. (1959). Studies on the biological properties of Mycobacteriophage. Amer. Rev. respir. Dis. 80, 543.

Uetake, H., Nakagawa, T. \& Akiba, T. (1955). The relationship of bacteriophage to antigenic changes in group $\mathrm{E}$ salmonellas. J. Bact. 69, 571.

Uetake, H., Luria, S. E. \& Burrows, J. W. (1958). Conversion of somatic antigens in Salmonella by phage infection leading to lysis or lysogeny. Virology, 5, 68.

White, A. \& KNight, V. (1958). Effect of Tween 80 and serum on the interaction of mycobacteriophage D-29 with certain mycobacterial species. Amer. Rev. Tuberc. 77, 134.

Zinder, N. D. (1957). Lysogenic conversion in Salmonella typhimurium. Science, 126, 1237.

\section{EXPLANATION OF PLATE}

The phage susceptible strain of chromogenic-acid fast bacteria and the phage-immune variant.

Fig. 1. Colonies of the phage-susceptible strain after 12 days of incubation at $37^{\circ}$.

Fig. 2. Colonies of the phage-immune variant after 5 days of incubation at $37^{\circ}$ in the dark.

Fig. 3. Colonies of the phage-immune variant after 5 days of incubation at $22^{\circ}$ in the light. 
Journal of General Microbiology, Vol. 24, No. 1

Plate 1

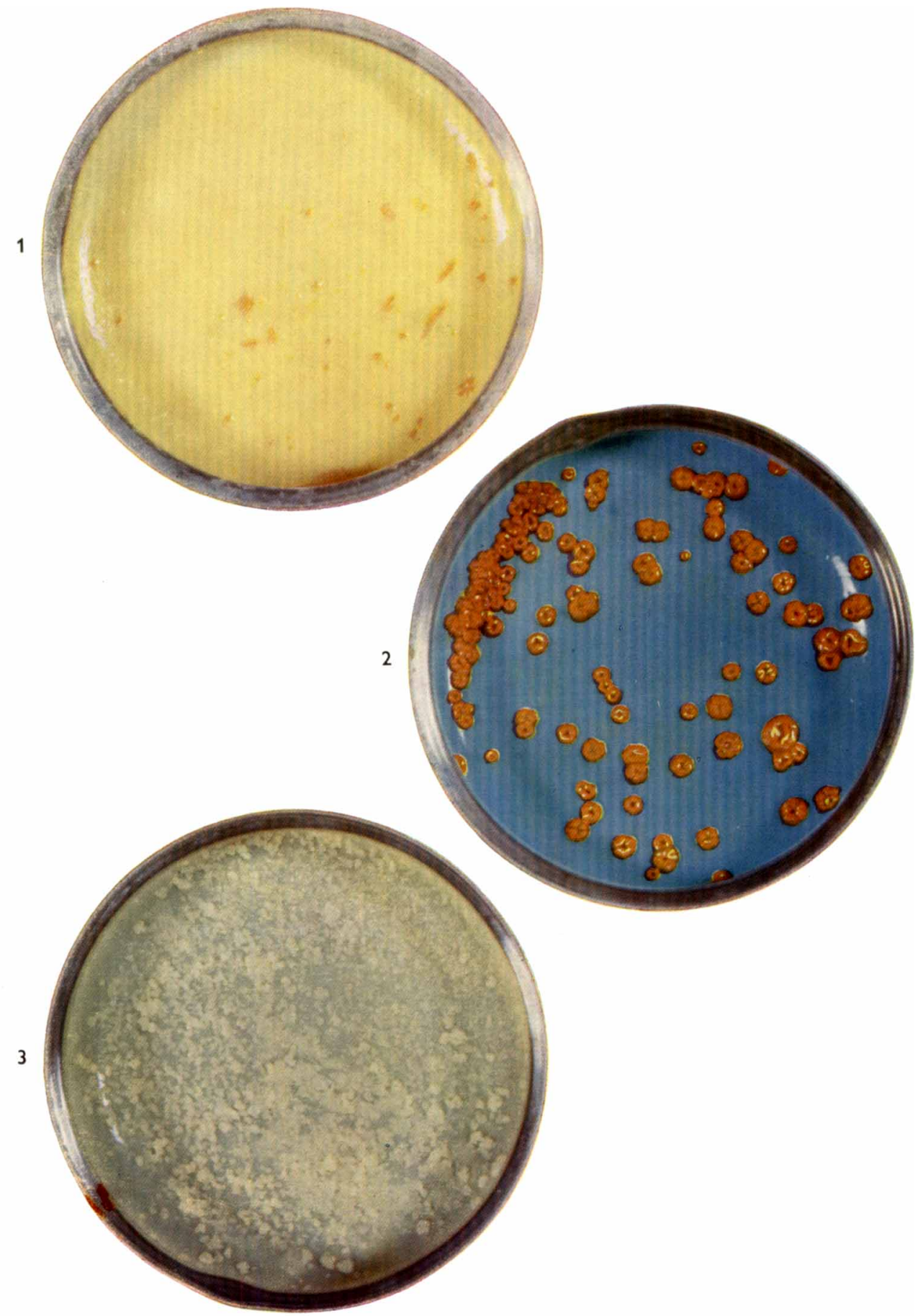

E. MANKIEWICZ

(Facing p. 68) 